\title{
The Response Activation Model and Cross-Modal Facilitation and Inhibition of Return: A Trajectory Analysis
}

\author{
Lawrence E.M. Grierson ${ }^{1}$, Timothy N. Welsh ${ }^{2}$, Steve Hansen ${ }^{1,3}$, Nicola J. Hodges ${ }^{4}$, Spencer Hayes ${ }^{3}$, \\ James Lyons ${ }^{1}$ and Digby Elliott ${ }^{1,3, *}$
}

\author{
${ }^{1}$ Department of Kinesiology, McMaster University, Hamilton, Ontario, Canada $;{ }^{2}$ Faculty of Kinesiology, Department of \\ Clinical Neurosciences, Faculty of Medicine, University of Calgary, Calgary, Alberta, Canada; ${ }^{3}$ School of Sport and \\ Exercise Sciences, Liverpool John Moores University, Liverpool, UK and ${ }^{4}$ School of Human Kinetics, University of Brit- \\ ish Columbia, British Columbia, Canada
}

\begin{abstract}
Non-informative spatial cues presented prior to a goal-directed movement influence not only movement initiation time but also the spatial characteristics of the movement trajectories. These trajectory effects are thought to stem from an integration of competing motor responses. In the present experiments, trajectories of rapid aiming movements were examined under the constraints of a cue-target inhibition of return (IOR) paradigm. Aiming movements were made to targets that were preceded by a cue stimulus in the same or different location. Four experiments were conducted in which the modality of the cue and target stimulus was manipulated across vision and audition. Although facilitation effects were present under the cross modality protocols, IOR effects were observed only for same cue-target pairings. At short stimulus onset asynchronies, limb trajectories deviated toward the target that had just been cued, particularly when the cue occurred in left space. These trajectory effects are consistent with response activation models of selective attention and movement preparation.
\end{abstract}

\section{INTRODUCTION}

The inhibition of return (IOR) phenomenon is thought to be an evolutionary adaptation designed to optimize human visual search behaviour, in that it hinders an individual's tendency to re-search already explored regions of visual space [1]. In the typical IOR study, target stimuli are presented at the same location as, or a different location from, an attention-capturing cue event. Reaction times (RTs) for responses to target stimuli presented at the same location as a preceding attention-capturing event are shorter than those to target stimuli appearing at other locations when the two events occur in close temporal proximity. Over time, however, this facilitation decays and an inhibitory effect emerges such that longer RTs are evident to target stimuli at the location of the previously presented cue [2].

Originally posited as a description of visual attentional capture, a considerable amount of research has been conducted on the IOR phenomenon and how it operates under other sensory modalities such as audition. Although the RT findings of these studies have not been consistent [3-7], they have prompted the suggestion that the initial response facilitation period lasts longer for auditory stimuli than visual stimuli and that auditory IOR takes more time to develop, as auditory information takes longer to reach the superior colliculus; a neural substrate considered important for unconscious oculormotor programming [8] and for prompting inhibition [3, 9-11]. Further it has been hypothesized that inhibition occurs later for auditory target responses because they are more difficult to spatially localize than visual targets [3].

*Address correspondence to this author at the School of Sport and Exercise Sciences, Liverpool John Moores University, Liverpool, England; Tel: 44 (0) 151231 4353; Fax: 44 (0) 151231 4037; E-mail: D.Elliott@ljmu.ac.uk
Although discrepancies in the literature may be related to differences in the processing speed of the modalities under consideration, a variety of different responses have been used in each of the previous experiments - from simple key press with the foot [6] to manual aiming responses [3]. Thus, it may also be the case that variations in the pattern of RT effects may be related to the different types of responses used. Although RT is an excellent index of movement preparation, it may not tell the whole story about any response competition that occurs as the movement unfolds.

Action-centred theories of attention are based on the premise that the perception and dedication of attention to a stimulus is concomitant with the organization of actions to interact with the stimulus object. This is achieved through excitation of shared spatial and motor mapping cortical systems and early activation of visual occipital areas for oculomotor responses [12-15]. Possible responses are excited and require independent inhibitory processes in order to suppress and eliminate those that are undesired because of this perception-action coupling. As an example, Welsh and Elliott [15] found that while competing targets had little impact on the time to initiate a movement, the actual reaching trajectory was influenced by the spatial location of the other stimulus event $[13,16,17]$. In terms of the IOR phenomenon, the latter action models suggest that the initial neuronal excitation is responsible for facilitation and the subsequent inhibition for the RT delays. Thus the current work was designed to extend previous multimodal studies of IOR by examining the kinematic characteristics of the limb trajectories.

In this set of four experiments, participants completed a series of movements to cued and uncued target locations. Across the four experiments we manipulated the sensorymodality of the cue and target such that they were either the 
same (i.e., visual-visual, auditory-auditory) or different (i.e., visual-auditory, auditory-visual). Within an experiment we manipulated stimulus onset asynchronies (SOA) to examine response facilitation and inhibition effects in both movement initiation and properties of the movement response. In order to accommodate the length of time needed to use auditory signals as the cue stimulus [3], our short SOA was set at 300 $\mathrm{ms}$ (which is approximately $75 \mathrm{~ms}$ longer than has been documented necessary to elicit facilitation effects with visual signals, [2]). The long SOA was $1200 \mathrm{~ms}$.

Of primary concern was how visual and auditory cues and targets at particular locations would elicit actions to acquire those locations. For example, if the link between attention and action is so tight that any capture of attention elicits a particular motor response irrespective of the sensory modality of the stimulus, then RT and trajectory effects should be similar across all cue-target modality pairings. That is, visual and auditory stimuli that sufficiently capture attention should evoke the same response activation and subsequently the same trajectory patterns. However, if these action affordances are specific to a modality, then the trajectory effects observed in one experimental condition might not be observed in the other conditions. For example, conditions that share identical cue stimuli (visual-visual and visual-auditory; auditory-auditory and auditory-visual) may elicit similar trajectory effects because the cue stimulus that precedes the imperative signal affords a similar response. Alternatively, response competition may only occur under congruent cuestimulus conditions (visual-visual, auditory-auditory).

\section{METHOD}

\section{Participants}

Forty right-handed individuals from the McMaster University community participated. The participants were assigned to one of the four experiments such that there were 10 ( 5 male, 5 female) individuals in each of the cue-target protocols. All procedures were conducted according to the ethical guidelines of McMaster University. Participants provided informed consent and all individual were awarded \$5 Cnd for their participation.

\section{Task and Apparatus}

Participants sat comfortably at an identical apparatus for each of the 4 experimental protocols. A red home position (2 $\mathrm{cm}$ diameter) was located directly in front and along the midline of the participants. Two target buttons $(2 \mathrm{~cm}$ diameter $)$ were mounted $17 \mathrm{~cm}$ further along the participant's midline and $17 \mathrm{~cm}$ to the left and the right of this midline, such that the limb needed to move approximately $24 \mathrm{~cm}$ to cover the distance from the home position to the target location. Positioned $3 \mathrm{~cm}$ behind each of these buttons was a small light ( 3 $\mathrm{cm}$ diameter). A speaker was positioned behind each of the lights. The lights and speakers provided visual and auditory cue and target stimuli. A black "+" served as a central fixation point and was placed directly between the two target buttons. Recording of RT, movement time (MT), and operation of the lights and speakers, was controlled using an Eprime program. Participants wore an infrared light emitting diode (IRED) on the index finger of their right hand. Position of the IRED was recorded at $500 \mathrm{~Hz}$ using an Optotrak 3020 .

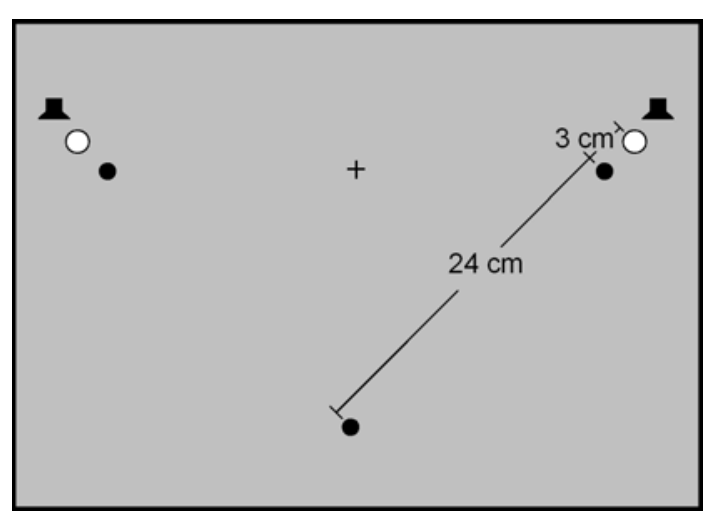

Fig. (1). Depiction of the experimental apparatus. The small dark circles represent the home position and the two targets and the + is the fixation point. The white circles represent the visual stimuli, with the speakers for the auditory stimuli right behind them.

\section{Procedure}

Each trial began with the participant sitting at the table with the right index finger depressing the home button. Their gaze was to be focused on the central fixation point. A 200 ms cue signal was presented at either the left or right target location. Participants were told to ignore the first stimulus (cue) and respond only to the second stimulus (target). After a variable interstimulus interval of either $100 \mathrm{~ms}$ or $1000 \mathrm{~ms}$, the target stimulus was presented to the participant. The target stimulus was presented in a counterbalanced and pseudorandom fashion at either the previously cued $(\mathrm{p}=.5)$ or un-cued location $(p=.5)$. The length of the cue and the interstimulus interval combined such that there was either a 300 ms or 1200 ms SOA between the cue and target stimuli. Participants were instructed to move as quickly and as accurately as possible to the location indicated by the target stimulus. Upon successful execution of the movement, participants heard a $20 \mathrm{~ms}$ centralized beep that signaled them return to the home position, re-fixate on the central fixation point, and prepare for the next trial. There was a minimum inter-trial interval of 2 seconds. In each experiment, participants completed 8 practice trials before completing the experimental trials that were presented in a random order. Participants completed 120 trials to the cued location and 120 trials to the un-cued location, for a total of 240 trials. Half the trials were completed with $300 \mathrm{~ms}$ SOA and the other half with $1200 \mathrm{~ms}$ with an equal number of left and right (cue $\mathrm{X}$ target) trials.

One experimental group was exposed to a series of trials in which both the cue and target were presented as visual signals $(3 \mathrm{~cm}$ red lights). Likewise, another group received both signals as auditory tones $(4.4 \mathrm{kHz})$. The two remaining groups received the stimulus across the two modalities; with one group seeing a visual cue and hearing an auditory target signal (visual-auditory) and the other hearing an auditory cue and seeing a visual target signal (auditory-visual). In all conditions the participants were aware of the stimulus modality of the cues and targets.

\section{Data Reduction and Analysis}

RT and MT were subjected to separate 2-Cue (left or right) by 2-Target (left or right) by 2-SOA (300 or $1200 \mathrm{~ms}$ ) 
repeated measures analysis of variance. The four cuestimulus protocols were treated as separate experiments. Three-dimensional IRED positions were filtered via a Butterworth filter with a cut-off frequency of $10 \mathrm{~Hz}$ to remove any nonbiological sampling artifacts. Resultant displacements were then differentiated once to obtain velocity and a second time to obtain acceleration. Custom software was applied to the displacement, velocity, and acceleration data to determine the spatial location in the horizontal direction (i.e., left-right) at peak acceleration, peak velocity, peak deceleration, and the end of the movement. These locations were expressed in terms of their absolute deviation from the midline (home position) and served to index any deviation toward or away from the cue. Due to an Optotrak data collection error in the visual-auditory protocol, one participant was removed from the analysis of kinematic deviation. The kinematic data were subjected to a similar analysis as RT and MT with the addition of a fourth factor pertaining to the kinematic landmark (i.e., peak acceleration, peak velocity, peak deceleration and movement endpoint). Significant main effects and interactions of interest were examined using Tukey HSD post hoc procedures $(p<.05)$. In particular we focused on the highest order interactions in view of the complex dependencies of cue, target and SOA.

\section{RESULTS AND DISCUSSION}

\section{Visual-Visual Protocol}

The analysis of RT in this protocol yielded main effects for SOA, $F(1,9)=7.10, p<.05$, and Target, $F(1,9)=15.26$, $p<.005$, and an interaction between SOA and Target, $F(1,9)$ $=13.99, p<.005$. Post hoc analysis revealed that responses made at the long SOA to the right target had the shortest RT and those made at the short SOA to the left target the longest RT. RT of movements made to the right target at the short SOA and to the left target at the long SOA were intermediate in duration and did not differ significantly from one another. The Cue by Target interaction was significant, $F(1,9)=$ $12.56, p<.01$. This interaction revealed a side of spacespecific IOR effect in that RTs were shorter in the cued-left, target-right pairing relative to the other cue- target pairings. Given our relatively long $(300 \mathrm{~ms})$ "short" SOA to make the durations comparable across protocol, it is not surprising that the three-way interaction between, Cue, Target, and SOA, $F(1,9)=.92, p<.36$ was not significant in the visual-visual protocol. A significant interaction of this type is typically indicative of facilitation and IOR effects, with the interaction being driven by the contrary differences in RTs made to previously cued target or previously un-cued targets that are

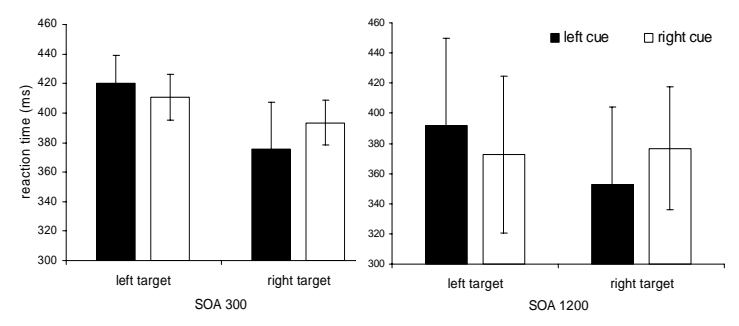

Fig. (2a). Reaction time (ms) as a function of cue (Left [black], Right [white]), target (left, right), and stimulus onset asynchrony $(300,1200)$ for the visual-visual condition. manifested at shorter and longer SOAs. However, in view of the relatively long SOAs for the visual stimuli in comparison to other studies, the short SOA showed a similar pattern to the longer SOA condition. This pattern is in line with the notion of a building inhibitory process to previously cued locations and is substantiated by post hoc results that indicate that at both the long and the short SOA, cued left, rightward movements are initiated earlier than cued left, leftward movements.

There were no facilitation or inhibition effects for MT, only a significant main effect for Target, $F(1,9)=88.12, p<$ .0001 . Movements made to the right target were of a shorter duration than those made to the left. This is a typical ipsilateral advantage associated with right-handed participants making right hand movements in right space [18].

Table 1. Movement Time Means (ms) for Each of the CueTarget Pairing Protocols (SOA Represents Stimulus Onset Asynchrony)

\begin{tabular}{|c|c|c|c|c|}
\hline \multirow{2}{*}{} & \multicolumn{2}{|c|}{ Left Target } & \multicolumn{2}{c|}{ Right Target } \\
\cline { 2 - 5 } & 300 ms SOA & $\mathbf{1 2 0 0}$ ms SOA & 300 ms SOA & $\mathbf{1 2 0 0}$ ms SOA \\
\hline \hline $\begin{array}{c}\text { Visual } \\
\text { Visual }\end{array}$ & 843.14 & 850.86 & 631.93 & 620.95 \\
\hline $\begin{array}{c}\text { Auditory } \\
\text { Auditory }\end{array}$ & 736.47 & 733.94 & 580.27 & 565.28 \\
\hline $\begin{array}{c}\text { Auditory } \\
\text { Visual }\end{array}$ & 906.83 & 905.02 & 710.82 & 704.45 \\
\hline $\begin{array}{c}\text { Visual } \\
\text { Auditory }\end{array}$ & 886.73 & 892.83 & 766.32 & 759.38 \\
\hline
\end{tabular}

Results of the analysis of variance of the kinematic deviations in the horizontal plane yielded main effects for Kinematic Marker, $F(3,27)=1169.84, p<.0001$, Cue, $F(1,9)=32.39, p<.0001$, Target, $F(1,9)=22.96, p<.0001$, and SOA, $F(1,9)=21.27, p<.01$. There were numerous two-way and three-way interactions that were superceded by a significant 4-way Kinematic Marker by Cue by Target by SOA interaction, $F(3,27)=16.06, p<.0001$. These data are illustrated in Fig. (3a).

Post hoc analysis of this interaction revealed that in addition to the increased horizontal deviation from the home position that occurs as the reach movement develops across kinematic marker, a noted difference was evident when movements were made towards the right target under the constraints of a left, rather than right cue and the short SOA. There was a marked reduction in the horizontal deviation from the home position at each of the kinematic markers after and including peak velocity in comparison to the other target and cue conditions. This reduced deviation is indicative of a veering motion towards the task irrelevant left target and can be interpreted as a pull that is created by the attentional processes related to this non-informative cue. Thus at $300 \mathrm{~ms}$, participants have not been able to completely disengage response activation associated with a left cue. Consistent with Tremblay, Welsh and Elliott [19], this finding 
could reflect the greater stability of left space activation effects.
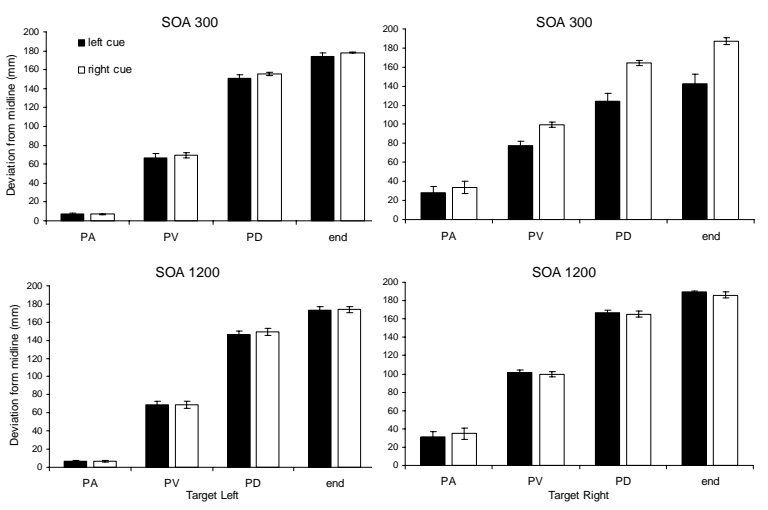

Fig. (3a). Deviation from the midline $(\mathrm{mm})$ as a function of cue (Left [black], Right [white]), stimulus onset asynchrony (300, 1200), target (left, right), and kinematic marker (peak acceleration $=$ pa, peak velocity $=p v$, peak deceleration $=p d$, movement endpoint $=$ end) for the visual-visual condition

\section{Auditory-Auditory Protocol}

RT main effects, in the auditory-auditory condition, were manifested for both Cue, $F(1,9)=6.20, p<.05$, and Target, $F(1,9)=13.45, p<.01$. A significant 2 -way SOA by Cue interaction, $F(1,9)=6.89, p<.05$ was also evident. Importantly, this protocol yielded a three-way SOA by Cue by Target interaction, $F(1,9)=75.39, p<.0001$, as illustrated in Fig. (2b).
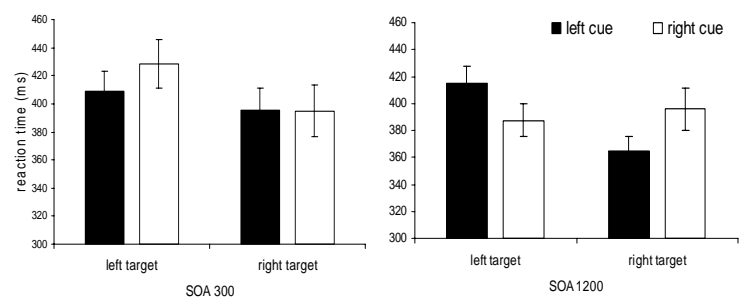

Fig. (2b). Reaction time (ms) as a function of cue (Left [black], Right [white]), target (left, right), and stimulus onset asynchrony $(300,1200)$ for the auditory-auditory condition.

Post hoc analysis of this interaction revealed that at the short SOA, responses made to left-cued, left targets were initiated earlier than those made to right-cued, left targets and right-cued, right target movements were initiated sooner than right-cued, left target movement. However, there was no difference between the left and right cued, right target conditions. These results are indicative of the facilitation, at least for the left target conditions. At the long SOAs, the contra-lateral cue-target pairing conditions were fastest, in comparison to the ipsilateral pairings, suggesting an inhibition at long SOAs for same cue and target locations.

Analysis of the MT data in this condition showed a significant main effect for Target, $F(1,9)=30.86, p<.001$. As was the case in the visual-visual condition, this effect represents the ipsilateral advantage for the right target (Table 1).

Analysis of the horizontal kinematic deviations revealed significant main effects for Kinematic Marker, $F(3,27)=$ $30.80, p<.0001$, Cue, $F(1,9)=40.80, p<.001$, Target, $F(1,9)=6.41, p<.05$, and SOA, $\mathrm{F}=12.89, p<.01$. Again, as was the case in the visual-visual protocol, the analysis of horizontal kinematic deviation revealed many significant two-way and three-way interactions that were superceded by a significant four-way Kinematic Marker by Cue by Target by SOA interaction, $F(3,27)=3.02$, p $<.05$. Thus the pattern of results in Fig. (3b) is very similar to those already seen in Fig. (3a).
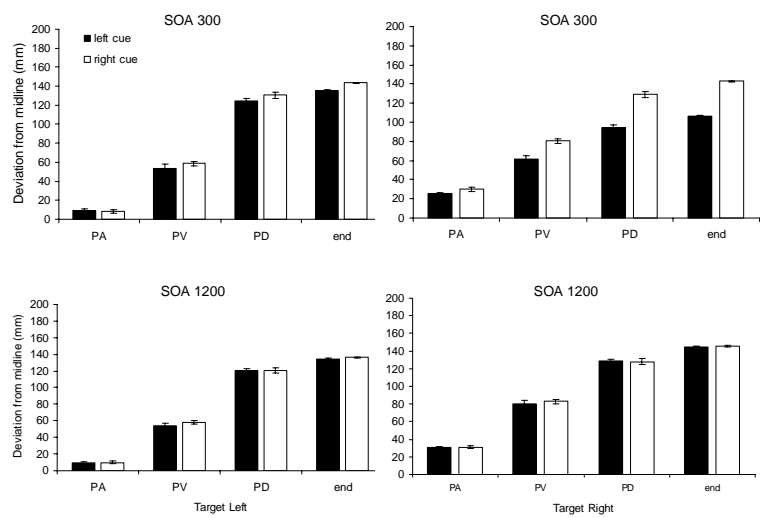

Fig. (3b). Deviation from the midline $(\mathrm{mm})$ as a function of cue (Left [black], Right [white]), stimulus onset asynchrony (300, 1200), target (left, right), and kinematic marker (peak acceleration $=$ pa, peak velocity $=$ pv, peak deceleration $=p d$, movement endpoint $=$ end) for the auditory-auditory condition.

The post hoc analysis again indicated that left-cued movements made to the right target at a short stimulus onset asynchrony exhibited a reduced horizontal deviation compared to all other combinations examined.

\section{Visual-Auditory Protocol}

For the visual-auditory protocol (see Fig. 2c), there was a main RT effect for Target, $F(1,9)=6.06, p<.05$, with responses made to the left target initiated earlier than those to the right. A Cue by Target interaction, $F(1,9)=5.31, p<.05$, was also evident. Post hoc analysis of this interaction indicates that left-cued, left target movements were initiated sooner than those made to a left-cued right target. Although the three-way interaction was not significant, $F(1,9)=4.09$, $\mathrm{p}<.073$, facilitation effects were observed at the short SOAs and no facilitation or inhibition of return effects were observed under the long SOA conditions. MT data once again elicited a Target main effect, $F(1,9)=14.11, p<.005$, that again represents the right target ipsilateral advantage (Table 1).

The horizontal kinematic analysis yielded a main effect for Kinematic Marker, $F(3,24)=31.25, p<.0001$, and Cue, $F(3,24)=7.42, p<.05$.

Consistent with the other conditions is the superceding four-way interaction, $F(3,24)=4.54, p<.05$ and again a very similar pattern of results (see Fig. 3c). 


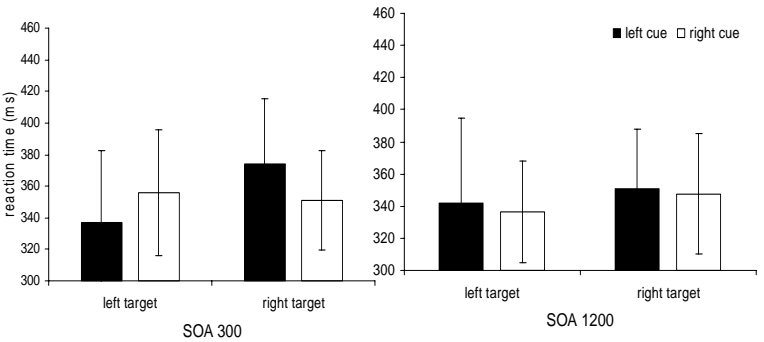

Fig. (2c). Reaction time (ms) as a function of cue (Left [black], Right [white]), target (left, right), and stimulus onset asynchrony $(300,1200)$ for the visual-auditory condition.

Movements made towards the left-cued, right target at the short stimulus onset asynchrony showed a decreased horizontal deviation from the centrally located home position. This increased pulling towards the non-informative left cue, coupled with the increased movement times to right located targets, may be a result of an increased effort to inhibit the effects of the task irrelevant left cue during the movement.
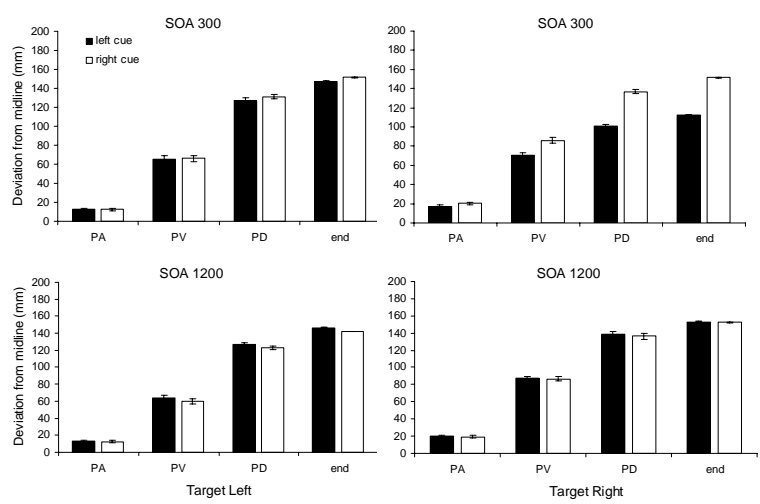

Fig. (3c). Deviation from the midline $(\mathrm{mm})$ as a function of cue (Left [black], Right [white]), stimulus onset asynchrony (300, 1200), target (left, right), and kinematic marker (peak acceleration $=$ pa, peak velocity $=p v$, peak deceleration $=p d$, movement endpoint $=$ end) for the visual-auditory condition.

\section{Auditory-Visual Protocol}

RT analysis revealed a significant 2-way Cue by Target interaction, $F(1,9)=12.01, p<.01$. Also revealed was a three way SOA by Cue by Target interaction, $F(1,9)=7.23, p<$ .05 , which is illustrated in Fig. (2d).
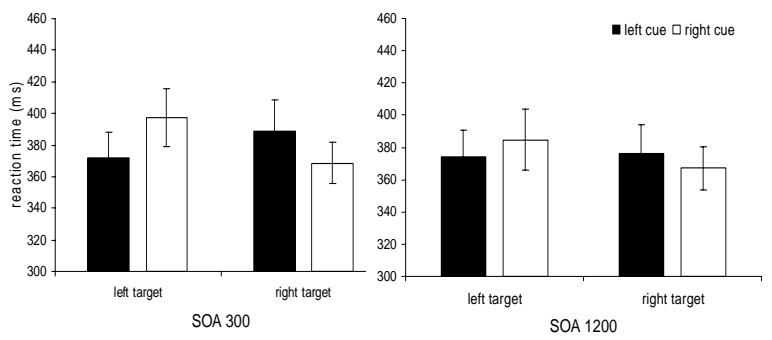

Fig. (2d). Reaction time (ms) as a function of cue (Left [black], Right [white]), target (left, right), and stimulus onset asynchrony $(300,1200)$ for the auditory-visual condition.
The interaction is not a result of the typical facilitation and inhibition at the short and long SOAs, respectively. Post hoc analysis revealed that the locus of this interaction effect was primarily manifested in the differences at the short SOA. Responses to same target and cue locations were initiated sooner than those to different cue-target locations. At the long SOA only shorter reaction times were observed for right-cued, right targets versus those made to right-cued, left targets. Individuals were facilitated by varying degrees when initiating movements to previously cued locations across SOA. No inhibitory processes were evident at either SOA.

Examination of the auditory visual protocol MT results yielded a main effect for Target, $F(1,9)=16.31, p<.005$. Movements made to the right target were completed quicker than those made to the left target (Table 1).
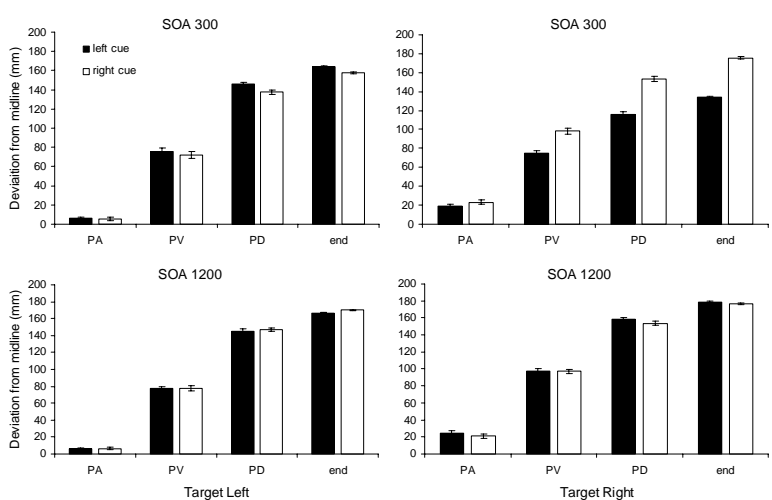

Fig. (3d). Deviation from the midline $(\mathrm{mm})$ as a function of cue (Left, Right), stimulus onset asynchrony (300, 1200), target (left, right), and kinematic marker (peak acceleration $=$ pa, peak velocity $=$ pv, peak deceleration $=p d$, movement endpoint $=$ end $)$ for the auditory-visual condition

Analysis of the auditory-visual protocol horizontal kinematic trajectories revealed significant main effects for Kinematic Marker, $F(3,27)=270.43, p<.0001$, Cue, $F(1,9)=$ $7.09, p<.05$, and SOA, $F(1,9)=19.86, p<.005$. In addition to numerous significant two-way and three-way interactions, the results yielded a significant four-way interaction, $F(3$, $27)=16.86, p<.0001$. As with the other 3 experimental protocols a similar pattern of results emerged (Fig. 3d) in that movements made to the right target following a left cue at a short stimulus onset asynchrony were marked by a reduced horizontal deviation from the home position indicating a veering towards the location of the non-informative leftsided cue.

\section{Across Protocol Deviation Effects}

The four-way interaction in all the cue-target protocols is driven primarily by deviations toward the left cue when participants were aiming to right-sided targets with a short cuetarget interval. As one would expect from other trajectory work [20], the magnitude of this bias increased between peak acceleration and peak deceleration and stabilized until the end of the movement. It would appear that, regardless of cue or target modality, events in left space automatically activated competing response tendencies that then dissipated over the next $900 \mathrm{~ms}$ (i.e., a SOA of 300 and $1200 \mathrm{~ms}$ ). Al- 
ternatively, participants may adopt a strategy of devoting greater attentional resources to left space because these movements are more difficult for right-handed individuals performing with the right hand. This latter explanation is consistent with the notion that advance preparation is often biased in favour of the worst-case scenario [21]. In this context, there was a clear ipsilateral advantage for movement time (i.e., the right hand performing in right space). In any event, it is of interest that asymmetric trajectory effects were evident even in the vision-vision protocol where the temporal data suggest that performers had already begun to inhibit left-sided cues at $300 \mathrm{~ms}$.

\section{SUMMARY AND CONCLUSIONS}

The primary purpose of this research was to determine if limb trajectory biases associated with competing response tendencies could be elicited when the competing responses were activated by stimuli from different modalities. The work was designed to provide insight into the processes associated with cross-modal facilitation and inhibition of return, as well as specific action-based accounts of selective attention [7,13,15,22]. It was hypothesized that should the motor response codes initiated by the attentional system be independent of the modality of cue stimulus presentation then the movement trajectories elicited under the different perceptual constraints of the experiment would not differ. Conversely, should the properties of the visual and auditory capture interact with the attentional system in such a way that they afford different motor responses, the trajectories of the observed movements would not exhibit a consistent pattern. Although we failed to see the type of veering away from a competing target location that one might expect in a situation in which that target location or movement to that target location had been inhibited [15,23], participants were biased when pointing to right-sided targets by a left-sided cue if the SOA was short. This bias occurred regardless of how the cue-target locations were signaled. According to Welsh and Elliott's [15,23] response activation model, these trajectory effects occur because the cues activate response codes for movements in that direction (see [24] for similar results with saccadic eye movements).

Of additional interest was the finding that biases toward non-target stimuli in left space were more robust than biases in right space. While this finding is consistent with previous research related to the special role played by the right hemisphere in selective attention [25-28], these trajectory effects could also reflect a feedforward strategic approach adopted by participants because limb control is more difficult in contralateral space [18]. In either context, cues in left space may elicit either more intense or longer lasting response tendencies than cues in right space $[3,18]$.

Although our trajectory effects were consistent across cue-target protocol, our temporal effects were not. Specifically, while both facilitation (short SOA) and inhibition (long SOA) was evident under auditory-auditory conditions only inhibition was present in the visual-visual protocol (short and long SOA) and only facilitation (short SOA) was evident for the cross-modal pairings. While, as stated previously, the within-modality differences in facilitation vs inhibition are probably the result of the relative processing times for locating a position in space on the basis of light and sound, it is intriguing that cross-modal pairings of cue and target resulted in facilitation without inhibition (but see also [29]). In this context, it may be that facilitation and inhibition are not opposite sides of the same attentional coin and are likely two independent processes [2]. For example, in a recent transcranial magnetic stimulation study, Welsh, Gonzalez, Lyons and Elliott [30] found that while stimulation of primary motor cortex areas associated with muscles required for target acquisition enhanced typical response facilitation effects at short SOAs (i.e., $100 \mathrm{~ms}$ ), no impact was evident on later inhibitory effects (SOA of $1000 \mathrm{~ms}$ ). This suggests neuronal excitation related to facilitation may be associated with change in the representation of action in the motor systems while inhibitory mechanisms are more upstream (e.g., perceptual/attentional in nature) and/or subcortical [8]. Certainly this latter suggestion is consistent with the finding that movement trajectories veered toward, rather than away from, competing targets. If facilitation is associated very automatic motor processes, this could also account of its independence from stimulus modality.

In summary, the results of this study indicate that noninformative visual and auditory cues automatically excite response tendencies that can impact both the time to initiate and the trajectory of subsequent movements. The blending of target and non-target response tendencies appears to be more motor than perceptual in nature, and these effects are most pronounced when the initial auditory or visual stimulation occurs in left space. While these facilitation results are consistent with the response activation model and trajectory effects that have been found in distractor paradigms [15], both our temporal and (lack of) trajectory findings indicate that inhibition of return may operate upstream from the motor system. This latter suggestion is consistent with recent TMS work from our lab [30]. It is also compatible with recent target-target IOR studies that indicate inhibitory processes transfer across effector systems (i.e., the two hands, [19]) and even separate nervous systems $[31,32]$.

\section{ACKNOWLEDGEMENTS}

This research was supported by the Natural Sciences and Engineering Research Council of Canada as well as the Canada Research Chair Program.

\section{REFERENCES}

[1] Klein RM, MacInnes WJ. Inhibition of return is a foraging facilitator in visual search. Psychol Sci 1999; 10: 346-352.

[2] Posner MI, Cohen Y. In: Bouma H, Bouwhuis DG, Ed. Components of visual orienting, Attention and performance X. Hillsdale, NJ: Lawrence Earlbaum. 1984; 531-556.

[3] Lyons J, Glazebrook CM, Keetch KM, Dhillon VP, Elliott D. Influence of endogenous and exogenous orientations of attention on inhibition of return in a cross-modal target-target aiming task. J Mot Behav 2006; 38: 219-228.

[4] Reuter-Lorenz PA, Jha AP, Rosenquist JN. What is inhibited by inhibition of return? J Exp Psych Hum Percept Perfor 1996; 22: 367-378.

[5] Spence C, Driver J. Auditory and audiovisual inhibition of return. Percept Psychophys 1998; 60: 125-139.

[6] Spence C, Lloyd D, McGlone F, Nicholls MER, Driver J. Inhibition of return is supramodal: A demonstration between all possible pairings of vision, touch, and audition. Exp Brain Res 2000; 134: 42-48.

[7] Welsh TN, Elliott D. Multimodal inhibition of return effects in adults with and without Down syndrome. Dev Neuropsychol 2004; 25: 281-297. 
[8] Galfano G, Betta E, Turatto M Inhibition of return in microsaccades. Exp Brain Res 2004; 159: 400-404

[9] Posner MI, Rafal RD, Choate LS, Vaughn J. Inhibition of return; neural basis and function. Cogn Neuropsychol 1985; 2: 211-228

[10] Rafal RD, Calabresi PA, Brennan CW, Sciolto TK. Saccade preparation inhibits reorienting to recently attended locations. J Exp Psych Hum Percept Perfor, 1989; 15: 673-685.

[11] Sapir A, Soroker N, Berger A, Henik A. Inhibition of return in spatial attention: direct evidence for collicular generation. Nat Neurosci 1999; 2: 1053-1054.

[12] Rizzolatti G, Riggio L, Sheliga BM. In: Umilta C, Moscovitch M. Ed, Space and selective attention, Attention and performance XV. Cambridge, MA, MIT Press 1994; 231-265.

[13] Tipper SP, Howard LA Houghton G. In Monsell S, Driver D Ed, Behavioural consequences of selection form neural population codes, Attention and performance XVIII. Cambridge, MA: MIT Press 1999; 223-245.

[14] Tucker M, Ellis R. On the relations between seen actions and components of potential actions. J Exp Psych Hum Percept Perfor 1998; 24: 830-846.

[15] Welsh TN, Elliott D. Movement trajectories in the presence of a distracting stimulus: evidence for a response activation model of selective reaching. Q J Exp Psychol 2004; 57A: 1031-1057.

[16] Chang E, Ro T. Inhibition of return in perception and action. Visual Cognition 2005; 1: 443-472.

[17] Howard LA, Lupianez J, Tipper SP. Inhibition of return in a selective reaching task: An investigation of reference frames. J Gen Psychol 1999; 126: 421-442.

[18] Elliott D, Roy EA, Goodman D, Carson R, Chua R, Maraj BKV. Asymmetries in the preparation and control of manual aiming movements. Can Journal Exp Psychol 1993; 47: 570-589.

[19] Tremblay L, Welsh TN, Elliott D. Between-trial inhibition and facilitation in goal-directed aiming: manual and spatial asymmetries. Exp Brain Res 2005; 160: 79-88.

[20] Hansen S, Elliott D, Tremblay L. On-line control of discrete action following visual perturbation. Perception 2007; 36: 268-287.
[21] Elliott D, Helsen WF, Chua R. A century later: Woodworth's (1899) two-component model of goal-directed aiming. Psychol Bull 2001; 127: 342-357.

[22] Welsh TN, Elliott D. The effects of response priming on the planning and execution of goal-directed movements in the presence of distracting stimulus. Acta Psychol 2005; 119: 123-142

[23] Welsh TN, Elliott D. The effects of response priming and inhibition on movement planning and execution. J Mot Behav 2004; 36: 200211

[24] Sheliga BM, Craighero L, Riggio L, Rizzolatti G. (1997). Effects of spatial attention on directional manual and ocular responses. Exp Brain Res 1997; 114: 339-351.

[25] Heilman KM, Valenstein E Ed. Neglect and related disorders, Clinical neuropsychology. New York: Oxford 1979; 268-307.

[26] Heilman KM, Van den Abel T. Right hemisphere dominance for mediating cerebral activation. Neuropsychologia 1979; 17: 315321.

[27] Hodges NJ, Lyons J, Cockell D, Reed A, Elliott, D. Hand, space and attentional asymmetries in goal-directed manual aiming. Cortex 1997; 33: 251-269.

[28] Marzi, CA In: Denes G, Pizzamiglio L, Ed. Neuropsychology of attention, Handbook of clinical and experimental neuropsychology. Hove: Psychology Press 1999; 509-524.

[29] McAuliffe J, Pratt J. The role of temporal and spatial factors in covert orienting of visual attention tasks. Psychol Res 2005; 64: 285-291.

[30] Welsh TN, Gonzalez C, Lyons J, Elliott D. The influence of transcranial magnetic stimulation of primary motor cortex on facilitation and inhibition of return. Proceedings from the Society for Neuroscience Annual Conference, Washington DC 2005.

[31] Welsh TN, Elliott D, Anson JG, Dhillon V, Weeks DJ, Lyons J, Chua R. Does Joe influence Fred's action? Evidence for an evolutionary account of inhibition of return. Neurosci Lett 2005; 385: 99-104.

[32] Welsh TN, Lyons J, Weeks DJ, Anson JG, Chua R, Elliott D.Within-and between-nervous system inhibition of return: $\mathrm{Ex}$ tending the role of the mirror neurons. Psychon Bull Rev 2007; 14: 950-956.

(C) Grierson et al.; Licensee Bentham Open.

This is an open access article distributed under the terms of the Creative Commons Attribution License (http://creativecommons.org/license/by/2.5/), which permits unrestrictive use, distribution, and reproduction in any medium, provided the original work is properly cited. 